\section{\$23. Velocity Distribution Functions and Reactivity of D-T Fusion Plasma with Spin-Polarized Fuel}

Takahashi, T. (Dept. Elect. Eng., Gunma Univ.), Nakao, Y. (Kyushu Univ.)

In 1982, Kulsrud et al. have examined the possibility of spin-polarized fusion and found that the depolarization rate of polarized fusion fuels is very small compared to the reaction rates in the case that the mechanisms of depolarization such as the binary collisions, the fluctuation of magnetic field and the inhomogeneity of the field are taken into account[1]. In the present paper, we have made the kinetic model with velocity distributions indexed by the magnetic quantum numbers for spin-polarized fusion plasmas and investigated the effect of depolarization of the polarized fuels on the thermonuclear reactivity for $\mathrm{D}$ - $\mathrm{T}$ fusion plasma.

Since the spin quantum number of deuteron $\mathrm{D}$ is unity, the magnetic quantum number of $\mathrm{D}, m_{\mathrm{D}}$, has the values of 1,0 and -1 . Deuterons $D$ in an unpolarized plasma equally exist in the three spin states. Thus the probabilities of the existence in each spin states become $1 / 3$. The probabilities for polarized particles, however, should not be identical among three spin states. Therefore, the ordinary distribution function of $\mathrm{D}$, i.e., $f_{\mathrm{D}}(v)$, will be described by the expression:

$$
f_{\mathrm{D}}(v)=f_{\mathrm{D}}{ }^{+}(v)+f_{\mathrm{D}}{ }^{0}(v)+f_{\mathrm{D}}{ }^{-}(v)
$$

where the superscript,+ 0 and - represent the magnetic quantum number for $m_{\mathrm{D}}=1,0$ and -1 , respectively. Similary to $\mathrm{D}$, the distribution function of $\mathrm{T}$ is also classable by the spin states. Since the spin quantum number of $T$ is $1 / 2$, the magnetic quantum numbers of $T$ are $1 / 2$ and $-1 / 2$. Thus the distribution function of $\mathrm{T}$ should be classified as

$$
f_{\mathrm{T}}(v)=f_{\mathrm{T}}^{+}(v)+f_{\mathrm{T}}^{-}(v)
$$

where the superscript + and - represent $m_{\mathrm{T}}=1 / 2$ and $m_{\mathrm{T}}=-1 / 2$. Here, we consider the transition of the spin states of polarized particles in fusion plasmas. Particles supplied into a plasma are assumed to be perfectly polarized in states $m_{\mathrm{D}}=1$ and $m_{\mathrm{T}}=1 / 2$. We are able to consider the fuel particle loss due to the $\mathrm{D}-\mathrm{T}$ fusion reaction. The particle and the energy loss are expressed by using the confinement time dependent on the velocity of particles. The model equation is given by:

$$
\begin{aligned}
\left(\frac{\partial f_{a}^{m}}{\partial t}\right)= & \left(\frac{\partial f_{a}^{m}}{\partial t}\right)_{c o u}+\frac{1}{v^{2}} \frac{\partial}{\partial v}\left[\frac{v^{3} f_{a}^{m}}{2 \tau_{E}(v)}\right] \\
& +S_{a}^{m}(v)-L_{a}^{m}(v) \\
& -\sum_{m}\left(\frac{\partial f_{a}^{m}}{\partial t}\right)_{m \rightarrow m^{\prime}}+\sum_{m}\left(\frac{\partial f_{a}^{m^{\prime}}}{\partial t}\right)_{m^{\prime} \rightarrow m} \\
& =0
\end{aligned}
$$

The first term of R. H. S. represents the Coulomb collision term. The second term describes the particle loss from unit volume in velocity space due to the heat conduction. The third and fourth term, i. e., $S_{a}^{m}(v)$ and $L_{a}^{m}(v)$, indicate the source and the loss of a species particles in real space due to the particle injection, the nuclear reaction and the convection of particles, respectively.
Since the fusion reaction cross-sections are different depending on the spin states of D and $\mathrm{T}$, it is neccesary to modify the description of the fusion reactivity. When deuterons with the magnetic quantum number $\lambda$ react with tritons which have $\mu$, the thermonuclear reactivity $\overrightarrow{\langle\sigma \nu}\rangle_{D T}{ }^{\lambda \mu}$ is expressed by

$\overrightarrow{\langle\alpha\rangle_{D T}}{ }^{\lambda \mu}=\frac{8 \pi^{2}}{n_{D} \eta_{T}} \int_{0}^{\infty} v_{D} f_{D}^{\lambda}\left(v_{D}\right) \int_{0}^{\infty} v_{T} f_{T}^{\mu}\left(v_{T}\right)\left(\int_{u}^{w} \varepsilon_{\lambda \mu} \nu_{r}^{2} \sigma\left(v_{r}\right) d v_{r}\right) \mathrm{d} v_{\mathrm{D}} \mathrm{d} v_{\mathrm{T}}$ where $w=v_{T}+v_{D}$ and $u=\left|v_{T}-v_{D}\right|$. Thus thermonuclear reactivity of spin-polarized D-T fusion plasmas becomes,

$$
\left.\overrightarrow{\langle\sigma v}\rangle_{D T}=\sum_{\lambda \mu t} \overrightarrow{\langle\sigma v}\right\rangle_{D T} \lambda^{\mu}
$$

The thermonuclear reactivity for a polarized plasma with no depolarization is 1.5 times as large as the one for unpolarized plassma. Due to the depolarization mechanisms in the plasma the reactivity decreases to some extent. We will calculate the decrement of the reactivity $\eta$ defined as

$$
\eta=\frac{\overrightarrow{\langle\sigma v\rangle_{D T}}-\overrightarrow{\langle\sigma v\rangle_{0}}}{\overline{\langle\sigma v\rangle_{0}}} \times 100
$$

where $\overrightarrow{\langle\sigma\rangle_{0}}$ denotes the reactivity for complete polarization. The decrement $\eta$ becomes $-33.3[\%]$ as the fuels depolarize completely.

Varying the external magnetic field $B_{0}$ and the amplitude of magnetic fluctuation $\delta B$, the effects of waves have been considered. The decrement of reactivity $\eta$ are shown in Fig. 1 in the external field $B_{0}=1,5$ and $10 \mathrm{~T}$, respectively. In all cases, when $\delta B$ is smaller than $10^{-5} \mathrm{~T}$, the polarization and the alignment are kept unity. On the other hand, the amplitude $\delta B$ become greater than $10^{-5} \mathrm{~T}$, however, the polarization become ineffective..

For the current drive and the heating, lower hybrid waves are applied, whose frequency exists in $\mathrm{GHz}$. If lower hybrid current drive (LHCD) and heating (LHH) are applied, the plasmas will be depolarized consequently.

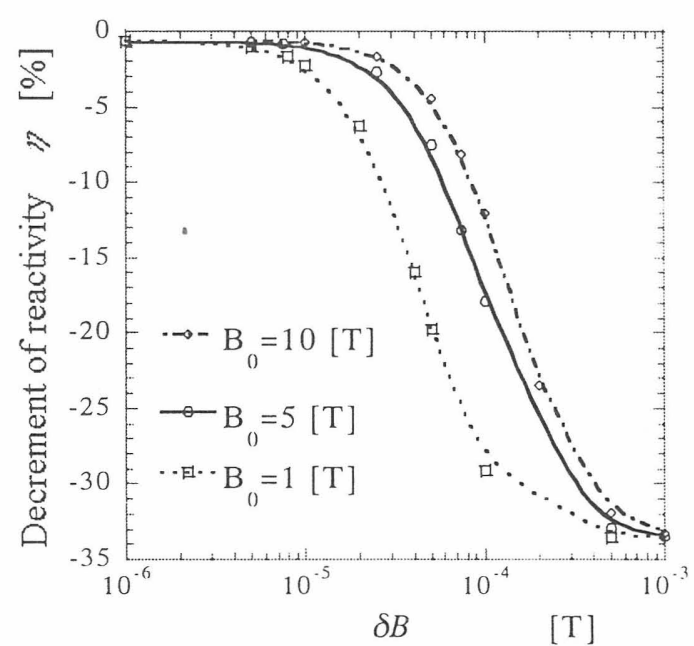

Fig.1 Decrement of reactivity $\eta$ versus the fluctuating magnetic field.

Reference

[1] R. M. Kulsrud et al., Phys. Rev. Lett. 49 (1982) 1248. 